\title{
La «realización científica» en la sociología de Colombia: una aproximación al estado de la disciplina*
}

\author{
Rodolfo Masías Nuñez \\ Carlos Aristizábal Botero*
}

Este artículo es resultado del programa de investigación «El investigador social en Colombia: producción, productividad, reconocimiento y celebridad".

** Doctor en Ciencias Sociales con especialidad en Sociología por El Colegio de México (México). Profesor asociado III de la Facultad de Ciencias Sociales de la Universidad de los Andes (Colombia). Correo electrónico: rmasias@uniandes. edu.co https://orcid.org/0000-0003-2512-6358

*** Candidato a doctor en Ciencias Sociales y Humanas de la Universidad Nacional de Colombia, sede Bogotá (Colombia). Profesor vinculado del programa de Sociología de la Universidad de Antioquia (Colombia). Correo electrónico: carlos.aristizabal@udea.edu.co https://orcid. org/0000-0001-5821-4878

Fecha de recepción: 15/08/2019. Fecha de aceptación: 21/04/2020 


\section{La «realización científica» en la sociología de Colombia: una aproximación al estado de la disciplina}

\section{RESUMEN}

Este estudio se aboca al conocimiento de la "realización científica» en la sociología colombiana. Son dos sus objetivos: analizar la realización científica como cuestión particular; y examinarla como una expresión del estado de la disciplina. Se busca complementar los «estados de la sociología» como aproximaciones globales, por lo general tendientes a observar la evolución de los temas de investigación y los desarrollos institucionales, pedagógicos y metodológicos en el campo de los sociólogos. «Realización científica» abarca la "producción» y "productividad» académica, pero como concepto forma parte de una perspectiva que las localiza en otra teoría, lo que faculta a una interpretación alternativa de estos mismos fenómenos.

El estudio se basa en un diseño muestral cuya unidad de análisis es el sociólogo o socióloga de las plantas académicas de las universidades con programas de sociología. Tiene como fuente las hojas de vida que los investigadores proveen al sistema nacional de la ciencia en Colombia (CVLAC). Ahí se halla toda su información académica y científica. Se presentan señales de un mundo sociológico fragmentado en términos de realización científica, como consecuencia de las grandes trasformaciones institucionales recientes. Esta fragmentación indica desigualdad entre los sociólogos. Una constatación así favorece la discusión sobre la constitución social de la sociología.

Palabras clave: estado de la disciplina, fragmentación académica, desigualdad académica, realización científica, productividad científica, sociología de la ciencia.

The «scientific achievement» in the sociology of Colombia: an approach to the state of the discipline

\section{Abstract}

This study focuses on the knowledge of «scientific achievement» in Colombian sociology. There are two objectives: to analyze scientific achievement as a particular matter; and examine as an expression of the state of discipline. It seeks to complement the "states of sociology" global approaches, generally tending to observe the evolution of research topics and institutional, pedagogical and methodological developments in the field of sociologists. "Scientific realization» encompasses academic "production» and "productivity", but as a concept it is part of a perspective that locates them in another theory, which enables an alternative interpretation of these same phenomena.

The study is based on a sample design whose unit of analysis is the sociologist from the academic plants of the universities with sociology programs. Its source is the resumes that researchers provide to the Colombian National Science System (CVLAC). There is all your academic and scientific information. Signs of a fragmented sociological world are presented in terms of scientific achievement, as a consequence of recent major.

Keywords: discipline status, academic fragmentation, academic inequality, scientific achievement, scientific productivity, sociology of science. 
En este artículo proponemos la perspectiva de la «realización científica» como un marco de análisis e interpretación especial para los estudios sociales de la ciencia (Latour, 2001, 2012 y Vink, 2015) y particularmente para procurar estados de una disciplina. La perspectiva representa «una expresión con la que $[. .$.$] se pretende des-$ pojar del sello productivista y mixtificador al cometido de consumar investigaciones y comunicarlas» (Masías, 2017). La enunciamos como una alternativa conceptual para abordar y abarcar los diferentes tipos de materialización y su posible comunicación, sin excepción, resultado de la actuación en la investigación social. En tal sentido, si se quiere como un modelo de investigación, su objetivo no es el de medir y valorar exclusivamente artículos científicos indexados, capítulos o libros, indicadores por excelencia de la llamada productividad científica, y sí el de explorar, conocer y articular otros tipos de realización, para luego indagar sobre sus vínculos con las condiciones sociales de los agentes que participan de un campo disciplinar. Nos interesa entender las formas de la realización y la sociabilidad que ellas propician en la relación del investigador con diferentes ámbitos sociales.

En esta perspectiva que proponemos, se entienden los comúnmente llamados «resultados» o "productos» de investigación como una concreción muy puntual de la incontable potencia creadora del sujeto investigador. Para decirlo en otras palabras, realización no es sinónimo de productos: la investigación no se agota en estas concreciones de la realización. Con esta conceptualización, los estudios de la consumación científica tendrían una alternativa más, en nuestra concepción con mayor poder de abstracción, para acceder al estudio de los mecanismos que gobiernan el conjunto de las prácticas emprendidas por los investigadores [«prácticas» en el sentido de Martínez y Huang (2015)], prácticas que expresan los fines de su acción para responder a los diferentes compromisos académicos de carácter institucional, así como a compromisos que, por su formación, considera propios de la labor de investigar en un ámbito disciplinar. Un beneficio de todo esto que venimos postulando como perspectiva de la realización es el de poder expresarse con más propiedad y contundencia del estado de una disciplina.

La realización científica es entendida, además, como un bien, patrimonio o capital de los investigadores, para hacer cierta analogía con el concepto de Bourdieu $(2003,2005)$. En la realización científica está contenido lo que cada investigador considera como quehacer investigativo, a lo que otorga crédito y lo que cree que lo posiciona mejor dentro del campo donde participa, según sea la estructuración de valores que en este se den. Con esta perspectiva es posible acceder a las marcas de diferenciación social entre los integrantes de las disciplinas que se estudien. Otra vez, los indicadores asociados a los modelos estandarizados de «resultados» o "productos» no deberían ser las marcas privilegiadas en el conocimiento de la vida social y política en la ciencia o la academia, pero consideramos aquí que la perspectiva 
que los relieva no permite percibir las condiciones de desigualdad e inequidad en el mundo de la investigación social, en el caso de este estudio, la sociología, para referirnos solo a esta realidad de la ciencia.

La realización supone que las materializaciones del investigador social sean valoradas en un mismo nivel. Para aclarar y acentuar, en esta perspectiva el artículo de investigación es visto en el mismo plano axiológico y con la misma importancia que otras materializaciones con las que se consuman procesos de investigación. Por esta razón, es necesario — para efectos del análisis — equiparar valorativamente cada forma de realización, de tal manera que, al compararlas, sea posible imaginar otras interpretaciones, clasificaciones, organizaciones, uso de medidas o demás de los intereses que orientan la acción de tales operaciones científicas. Al aplicar tal equivalencia, se hacen más visibles ciertas formas de acción investigativa que rara vez se tienen en cuenta, como ocurre con la visión productivista que hegemoniza. Con esta visión, es posible conocer el potencial de realización que las ciencias sociales tienen como empresa cognitiva. No obstante, para evidenciar todo esto, se recurre por ahora a las fuentes de información disponibles, con el ánimo de continuar construyendo los indicadores que de mejor manera permitan el abordaje de la realización.

En particular, consideramos que nuestra perspectiva puede aportar al conocimiento del estado de una disciplina, en el caso específico de la sociología, máxime en las condiciones sociohistóricas actuales, cuando el trabajo intelectual se precariza, como lo presenta Gill (2014). Así, conviene saber cuál es el despliegue de una disciplina, su constitución social, los rasgos del orden que la rige y los fenómenos de estratificación y desigualdad que presenta, asuntos de enorme trascendencia cuyo conocimiento favorece la intervención y el cambio. En efecto, una aproximación desde la realización complementa los estudios realizados sobre el estado de la sociología en Colombia: estudios globales, centrados en la evolución de los temas de investigación y los desarrollos institucionales, pedagógicos y metodológicos (Segura \& Camacho, 1999; Camacho \& Hernández, 1990; Cubides, 1998, 2011).

Para finalizar, este trabajo presenta mediciones de indicadores tradicionales de la realización científica que se usan para establecer la producción y productividad académica, como la publicación de artículos, de capítulos de libro y libros por cada uno de los sociólogos, pero también presenta mediciones de otros indicadores menos utilizados, aunque igualmente reveladores, como la cantidad total de resultados conseguidos por los investigadores, el promedio de artículos, capítulos y libros por año, el número de artículos publicados en revistas académicas, las publicaciones después de conseguir el doctorado y las revistas de publicación, entre otros. Si bien mostramos los resultados y los describimos, también ensayamos explicaciones para estos hallazgos enmarcadas en la perspectiva propuesta. No obstante, 
como habíamos anunciado antes, un propósito igualmente importante de este escrito es apuntar al estado de la sociología en Colombia. En consecuencia, leemos tales indicadores como aspectos de la realización de la sociología como un colectivo, comunidad o, si se quiere, sociedad, que (re)produce fenómenos de integración/ desintegración social, desigualdad, institucionalización de prácticas, conformación de jerarquías sociales y poderes políticos. Si bien un objetivo es dar con la realidad de la realización en sociología, otro es tomar la realización como signo de la constitución y estructuración social de esta disciplina apreciada como un microcosmos.

En la sección que sigue, exponemos la metodología de la investigación. Consta ahí el tipo de fuente utilizada y las razones de su elección, así como el alcance inferencial de los datos creados. Se presenta también el diseño muestral. Sin contar las conclusiones, las secciones posteriores contienen análisis de los hallazgos. A la descripción e interpretación de los resultados obtenidos por cada uno de los indicadores de realización elegidos, le sucede un análisis final de tipo explicativo. Un patrón inquietante que se observa en los hallazgos, y que es toda una conclusión preliminar que incita a más investigación, es la existencia, sea como se le quiera denominar, de una gran fragmentación, disparidad o desigualdad de realización científica entre los individuos considerados.

\section{Metodología}

Este estudio es otro logro del programa de investigación «El investigador social en Colombia: producción, productividad, reconocimiento y celebridad", que data de junio de 2010. Durante estos años, el programa ha desarrollado investigaciones del mismo corte que esta, sobre otras ciencias sociales o de las mismas ciencias sociales colombianas en conjunto. En principio, en cada caso nuevo que abarca, el programa replica, con fines comparativos, un mismo marco interpretativo y un mismo diseño metodológico, aplicados a las diferentes investigaciones realizadas (Masías, 2014, 2017). Asimismo, plasmadas estas investigaciones en artículos, el programa replica una misma secuencia narrativa y una misma definición de conceptos e indicadores.

A esta investigación le correspondió la elaboración de una propia base de datos, denominada «El investigador social de sociología _CVLAC». Ya se ha explicado (Masías, 2014, 2017) que el interés mayor de estas investigaciones no es hacer mediciones por sí mismas, ni tampoco insertarse en la línea de los llamados estudios bibliométricos; sino, dentro de la perspectiva de la realización científica, sentar indicios o evidencias que permitan entender y reflexionar más sobre la trascendencia de las enormes transformaciones que el mundo de las ciencias sociales ha venido experimentando en los últimos años (De Sousa Santos, 2003, 2010; Garcia Canclini, 2010). En la tabla 1 puede apreciarse el campo amplio de cuestiones e indicadores 
que abarca la base de datos, así como el conjunto de dimensiones operacionalizadas, entre otras, que componen el concepto de realización científica. En este trabajo presentamos resultados de solo alguno de estos indicadores.

Tabla 1. Variables de realización científica en la base de datos «El investigador social de sociología_CVLAC»

\begin{tabular}{|c|c|}
\hline \multicolumn{2}{|c|}{ Variable } \\
\hline Año de la primera experiencia docente & Número de cursos dictados \\
\hline Años de estudio hasta culminar el doctorado & Número de libros publicados \\
\hline Años de experiencia en cargo académico & Número de libros publicados: dicotomía \\
\hline $\begin{array}{l}\text { Años de servicio institucional (estabilidad } \\
\text { institucional) }\end{array}$ & Número de libros publicados: intervalos \\
\hline Años en cargos académicos administrativos & Número de productos en total \\
\hline Áreas de actuación & Número de productos en total: intervalos \\
\hline Carácter de la formación académica & $\begin{array}{l}\text { Número de publicaciones después del } \\
\text { doctorado }\end{array}$ \\
\hline $\begin{array}{l}\text { Carácter de la institución de los estudios de } \\
\text { pregrado }\end{array}$ & $\begin{array}{l}\text { Número de publicaciones después del } \\
\text { doctorado: intervalos }\end{array}$ \\
\hline $\begin{array}{l}\text { Características de la locación institucional } \\
\text { del investigador }\end{array}$ & $\begin{array}{l}\text { Número de revistas distintas de publicación: } \\
\text { dicotomía }\end{array}$ \\
\hline Ciudad de residencia o trabajo & $\begin{array}{l}\text { Número de revistas distintas de publicación: } \\
\text { intervalos }\end{array}$ \\
\hline $\begin{array}{l}\text { Ciudad de residencia o trabajo: Bogotá y } \\
\text { resto del país }\end{array}$ & Número de revistas distintas de publicación \\
\hline $\begin{array}{l}\text { Consistencia de la afiliación laboral del } \\
\text { investigador }\end{array}$ & Número total de productos: dicotomía \\
\hline Disciplina del investigador & País de doctorado \\
\hline $\begin{array}{l}\text { Disciplina del investigador: historia y no } \\
\text { historia }\end{array}$ & País de doctorado: Estados Unidos \\
\hline $\begin{array}{l}\text { Disciplina del investigador: por tradiciones } \\
\text { epistemológicas }\end{array}$ & País de maestría \\
\hline Edad & País de maestría: Colombia \\
\hline Edad: dicotomía & País de pregrado \\
\hline Escalafón profesoral & Persistencia académica del investigador \\
\hline Espacio predominante de publicación & Pertenencia a asociaciones académicas \\
\hline Género & Posesión de doctorado \\
\hline Grado de reputación & Posición de prestigio \\
\hline Grado de visibilidad & Posición institucional: decano \\
\hline Institución académica de trabajo & Posición institucional: director \\
\hline
\end{tabular}




\begin{tabular}{|c|c|}
\hline \multicolumn{2}{|c|}{ Variable } \\
\hline Institución de estudios de doctorado & Posición institucional: rector \\
\hline Institución de estudios de maestría & Premios obtenidos \\
\hline Institución de estudios de pregrado & Promedio de artículos por año \\
\hline $\begin{array}{l}\text { Institución de estudios de pregrado: mejor } \\
\text { universidad }\end{array}$ & Promedio de artículos por ańo: dicotomía \\
\hline Institución de estudios de pregrado: top & Promedio de artículos por año: intervalo \\
\hline $\begin{array}{l}\text { Institución académica de trabajo: universidad } \\
\text { top }\end{array}$ & Promedio de libros por año \\
\hline Líneas de investigación activas & Promedio de libros por año: dicotomía \\
\hline Líneas de investigación declaradas & Promedio de libros por ańo: intervalo \\
\hline Membrecías honoríficas & Proyectos consignados \\
\hline Número de afiliaciones académicas foráneas & Publicación temprana \\
\hline Número de artículos publicados & Reconocimiento temprano \\
\hline Número de artículos publicados: dicotomía & Región intelectual: doctorado \\
\hline Número de artículos publicados: intervalo & Región intelectual: maestría \\
\hline Número de capítulos de libros publicados & Selectividad de la universidad de origen \\
\hline $\begin{array}{l}\text { Número de capítulos de libros publicados: } \\
\text { intervalos }\end{array}$ & Selectividad del posgrado de origen \\
\hline Número de capítulos de libros: dicotomía & \\
\hline
\end{tabular}

El «individuo investigador» ha sido la unidad de análisis de las investigaciones del programa. En esta ocasión es aquel que forma parte de la sociología, en su condición de trabajador de alguno de los programas universitarios existentes en el país y, además, porque es un «investigador activo» en Colciencias ${ }^{1}$. Este es también un estudio muestral. La muestra fue definida a comienzos de 2017, momento en el cual contamos una población de 163 de estos sujetos, repartidos en nueve programas (18,11 individuos por programa en promedio). Esta cifra representa la planta universitaria de la sociología en Colombia. La muestra tiene un tamaño de 129 individuos, obtenida aplicando un margen de error de 4\% y un $95 \%$ de nivel de confianza. Con la muestra, se procedió luego a elegir aleatoriamente los casos. La idea era tomar sujetos de todos los programas existentes en el país; no obstante, no todos estos programas exponen o toda o alguna información sobre su planta.

\footnotetext{
1 «Colciencias es el Departamento Administrativo de Ciencia, Tecnología e Innovación. Promueve las políticas públicas para fomentar la CTI en Colombia. Las actividades alrededor del cumplimiento de su misión implican concertar políticas de fomento a la producción de conocimientos, construir capacidades para CTI, y propiciar la circulación y usos de estos para el desarrollo integral del país y el bienestar de los colombianos» (Colciencias, 2019b).
} 
Esos programas fueron excluidos. De modo que la muestra efectiva está conformada por programas de Bogotá, Cali, Medellín, Manizales y Barranquilla; con todo, una muestra bastante representativa del conjunto. La información de cada sujeto fue tomada de las hojas de vida CvLAC ${ }^{2}$. La base de datos contiene información actualizada y consolidada a enero de 2018 , mes que conforma la fecha de cierre de la base, pero que también representa el corte metodológico de la observación realizada, es decir, la «actualidad» que aborda el estudio, al ser una investigación sincrónica o de corte transversal. Se procesó la información con SPSS.

Estas son algunas características de la muestra: el 42,2\% es de Bogotá; 20,9\% de Cali; $15,5 \%$ de Medellín; 12,4\% de Manizales y 12,4\% de Barranquilla. 58,9\% son hombres y el $41,1 \%$ mujeres. $60,7 \%$ pertenecen a universidades públicas. Los programas de los que no pudimos conseguir información corresponden a Pasto, Buenaventura y César.

Bastante se ha especulado y debatido sobre la confiabilidad de la información existente en las hojas de vida CvLAC. Nosotros no desconocemos las críticas habidas ni los llamados atinados a ampliar el campo de asuntos que este formato contiene, para consignar una más cabal y mejor representada biografía académica (Forero, 2015; Navarrete, 2015). Ha trascendido que algunos investigadores no completan la información, que la abultan, y que otros no logran comprender lo solicitado. También se conoce de investigadores que han renunciado, por principios, a formar parte de este sistema. Sabemos, sin embargo, que las disposiciones de las instituciones académicas (universidades y centros de investigación) sobre utilizar obligatoriamente este sistema han tenido un efecto positivo, con lo que masivamente los investigadores de Colombia han entregado la información solicitada. Este estudio toma precauciones: sabe que debe expresarse en un tono menos categórico y quizás más conjetural. Pero está muy claro que esta información tiene un gran potencial que debiera usarse más y no despreciarse.

Asimismo, es necesario decir que, para una perspectiva de realización científica, esta fuente se convierte en el surtidor de información disponible estandarizada más completo que se posee, en tanto comprende diferentes indicadores de tipos de realización y no se circunscribe a los artículos y libros indexados. Esto hace factible un seguimiento más cabal a las acciones, prácticas y estrategias de realización científica

\footnotetext{
2 «Es el instrumento o formato electrónico de hoja de vida básico utilizado por Colciencias para el registro permanente de hojas de vida de investigadores, innovadores, académicos y expertos del SNCyTI. El registro de hojas de vida de candidatos a ingresar al servicio de información de pares evaluadores reconocidos del SNCyTI. Las convocatorias para reconocimiento y medición de Grupos Colombianos de Investigación Científica o Tecnológica» (Colciencias, 2019). Se trata de un aplicativo autodiligenciado por los investigadores de forma voluntaria. Los datos se encuentran disponibles en el sistema de información científico colombiano denominado SCIENTI.
} 
de los investigadores ${ }^{3}$. Un buen ejemplo de esto son los resultados de investigación que aquí mostramos y que resultan muy consistentes con los de los estudios anteriores, similares, que hemos ejecutado.

\section{LA SUERTE DE LA «REALIZACIÓN CIENTÍFICA» EN LA SOCIOLOGÍA}

Como ya se explicó en esta — como en las investigaciones anteriores (Masías, 2012, 2013, 2014, 2017)—, «realización científica» representa una perspectiva con que se pretende despojar de un monismo productivista el propósito de consumar investigaciones y comunicarlas. No obstante, al referirse a un conjunto de prácticas del mundo de la ciencia y la academia, representa formas de ser investigador, sociólogo o académico, según se trate. También refiere a concepciones de investigación e investigador y permite dar cuenta de las orientaciones realizativas de los sujetos en cuestión (Lowy, 2000).

Teniendo en mente la perspectiva de realización, la tabla 2 presenta información de varios de indicadores. Espacio predominante de publicación informa de la razón entre el número de revistas no científicas y científicas en que han publicado los sociólogos. Con ella se desvelan aspectos tanto de la identidad de investigador, como de investigador científico (una forma más específica de ser investigador). Para este indicador, razones cercanas a cero hablan de conductas realizativas definidas en términos del ámbito ideal de comunicación de los resultados del trabajo intelectual del sociólogo. El promedio sugiere que, en la sociología, sus investigadores tenderían a publicar más en revistas científicas, aunque sería una tendencia algo dispar en el conjunto, según se mire la desviación estándar, 0,81 . Por contraste a lo observado en otras ciencias sociales, y solo según este indicador, habría un predominio marcado de una realización investigativa de tipo científico en el campo de la sociología. La tabla 3 no hace sino solidificar esta interpretación: ahí se ve que un amplio sector de la muestra publica más en revistas científicas: razones de hasta 0,20 constituyen el $72,7 \%$ del total. De todos modos, no es despreciable la constatación de otros proyectos de realización, por así decirlo, sobre los cuales hay ausencia de información que permitiría comprender e identificar otros ámbitos de actuación del investigador social.

\footnotetext{
3 Desde un punto de vista metodológico, el programa no restringe sus investigaciones a una metodología cuantitativa. Es más, se es consciente de la importancia de conjugar metodologías y distintas fuentes de información para dar cuenta de los fenómenos de realización científica.
} 
Tabla 2. Variables descriptivas de realización científica

\begin{tabular}{lccc}
\hline & N & Media & Desviación \\
\hline Espacio predominante de publicación & 123 & 0,3060 & 0,81262 \\
Número productos en total & 126 & 17,45 & 19,498 \\
$\begin{array}{l}\text { Número de artículos publicados en revistas } \\
\text { académicas }\end{array}$ & 127 & 10,02 & 12,040 \\
Promedio de artículos por ańo & 125 & 0,9696 & 1,63884 \\
Revistas distintas de publicación & 126 & 6,48 & 6,575 \\
Número de capítulos de libros publicados & 126 & 4,90 & 7,068 \\
Número de libros publicados & 126 & 2,67 & 3,519 \\
Promedio de libros por ańo & 126 & 0,3690 & 0,49421 \\
Número de publicaciones después del & 85 & 9,79 & 14,113 \\
doctorado & & & \\
\hline
\end{tabular}

Tabla 3. Espacio predominante de publicación

\begin{tabular}{lcccc}
\hline & Frecuencia & Porcentaje & Porcentaje válido & Porcentaje acumulado \\
\hline $0,00-0,10$ & 33 & 25,6 & 60,0 & 60,0 \\
$0,11-0,20$ & 7 & 5,4 & 12,7 & 72,7 \\
$0,21-0,30$ & 1 & 0,8 & 1,8 & 74,5 \\
$0,31-0,40$ & 4 & 3,1 & 7,3 & 81,8 \\
$0,41-0,50$ & 2 & 1,6 & 3,6 & 85,5 \\
$0,51-$ HI & 8 & 6,2 & 14,5 & 100,0 \\
ND/SI & 74 & 57,4 & & \\
\hline Total & 55 & 42,6 & 100,0 & \\
\hline Total & 129 & 100,0 & & \\
\hline
\end{tabular}

Tal como se ha operacionalizado, el indicador Productos en total se obtiene de sumar el número de libros, de artículos y capítulos publicados por el sociólogo hasta el momento de cierre de la base de datos. Es un indicador útil si es visto con una visión sincrónica; de otro modo, puede dar la sensación de manifestar una medición falaz. Productos en total, en efecto, solo representa lo que, al momento del cierre del levantamiento de información, tiene en su haber cada sociólogo de los observados. Desde un interés sociológico, representaría lo que, en un punto específico del tiempo, en una actualidad puntual, coexiste en una comunidad académica.

Este indicador expresaría el tópico, en los estudios de la ciencia, que se manifiesta en tomarlo como una medida de "producción académica», tópico que se torna equívoco cuando en el sentido común de las ciencias sociales equivale a "productividad del investigador». En nuestra perspectiva (Masías, 2017), este indicador permite 
una aproximación a lo que consideramos sería la «obra» del investigador, algo más comprehensivo que todos los artículos o los libros publicados, lo que se acerca mucho a la noción de capital académico (Bourdieu, 2003, 2005). Este indicador representaría la capacidad del investigador para exteriorizar su potencialidad creativa presente en su actividad investigativa y académica. La tabla 2 muestra el promedio de productos en total por sociólogo, que es de 17,45, promedio no representativo si se considera la inmensa desviación estándar de 19,498. Adicionalmente, lo que se observa en la tabla 4 es una importante cantidad de investigadores con muy poca cantidad de realizaciones o productos en total. Entre 55\% y 69,8\% tendría una obra menor al promedio, por lo que habría muchos sujetos con poco o muy poco capital en los estándares del microcosmos de la sociología.

Tabla 4. Productos en total

\begin{tabular}{lcccc}
\hline & Frecuencia & Porcentaje & Porcentaje válido & Porcentaje acumulado \\
\hline Hasta 5 & 34 & 26,4 & 26,4 & 26,4 \\
$6-10$ & 29 & 22,5 & 22,5 & 48,8 \\
$11-15$ & 8 & 6,2 & 6,2 & 55,0 \\
$16-20$ & 19 & 14,7 & 14,7 & 69,8 \\
$21-25$ & 7 & 5,4 & 5,4 & 75,2 \\
$26-30$ & 12 & 9,3 & 9,3 & 84,5 \\
De 31 a más & 20 & 15,5 & 15,5 & 100,0 \\
\hline Total & 129 & 100,0 & 100,0 & \\
\hline
\end{tabular}

Hemos afirmado en otro lugar que, en la actualidad, centrar o abarcar la realización científica en la publicación de artículos se ha convertido en sinónimo de ser un investigador auténtico. Sin embargo, más auténtico y reconocible se es como investigador si las acciones realizativas se concentran en una creciente confección de artículos. No importa tanto qué otras realizaciones se emprendan, sino cuántos artículos se haya difundido y se esté planeando publicar. Publicar artículos es la forma de realización que expresa la mentalidad hacia la que más se insta por las institucionalidades científicas y académicas cuando se trata de definir lo que el científico social debe rendir como resultado de su labor. Como hemos señalado (Masías, 2013 y 2014), la cantidad de artículos publicados en revistas académicas tiende a ser vista, en el discurso científico dominante, como un indicador de la virtuosa propagación en una disciplina, de los valores y tipo de identidad científica que se relieva —un investigador con virtudes de productor empresarial eficiente y altamente racional-, pero también habla de la institucionalización de una práctica realizativa particular, como confeccionar artículos. 
El análisis global de lo concerniente a los artículos en una disciplina es clave y sumamente sugestivo. La tabla 2 contiene un promedio de 10,2 artículos publicados y una desviación de 12,04, dispersión otra vez muy relevante, un promedio muy poco central. Esta impresión se depura con la tabla 5, que contiene una tendencia ya identificada en otras disciplinas. Hay grandes realizadores de artículos en sociología, pero relativamente muy pocos, escasos. Particularmente, es preciso subrayar que $66,9 \%$ haya publicado hasta diez artículos y que el $48,8 \%$ solo cuente en su posesión hasta con cinco artículos.

Tabla 5. Artículos publicados en revistas académicas

\begin{tabular}{lcccc}
\hline & Frecuencia & Porcentaje & Porcentaje válido & Porcentaje acumulado \\
\hline 0 a 5 & 62 & 48,1 & 48,8 & 48,8 \\
$6-10$ & 23 & 17,8 & 18,1 & 66,9 \\
$11-15$ & 15 & 11,6 & 11,8 & 78,7 \\
$16-20$ & 11 & 8,5 & 8,7 & 87,4 \\
$21-25$ & 7 & 5,4 & 5,5 & 92,9 \\
$26-30$ & 2 & 1,6 & 1,6 & 94,5 \\
31 a más & 7 & 5,4 & 5,5 & 100,0 \\
No hay dato & 2 & 1,6 & & \\
\hline Total & 129 & 100,0 & & \\
\hline
\end{tabular}

Contra la manera como suele entenderse, el número de artículos publicados en revistas académicas no podría tomarse como un indicador especial de productividad (productividad de realización), porque no encarna la intensidad de la producción en el tiempo. Para nosotros, en cambio, el indicador promedio de artículos por año comprende esa dimensión (de tratarse de seguir el lenguaje y la perspectiva dominante en los estudios sobre el rendimiento científico). En la tabla 2, se ve una media de aproximadamente 0,969 artículos por año (menos de un artículo), en un contexto de una situación de gran desigualdad (una desviación estándar de 1,638). La tabla 6 comunicaría de forma viva la situación de la llamada «productividad académica» en sociología, ya que $40,8 \%$ de los investigadores solo llegaría a publicar hasta medio artículo por año, 55,2\% hasta tres cuartos de artículo y 75\% hasta un artículo por año. Respecto de la supuesta identidad ideal que considera que ser productivo en artículos es la forma de ser investigador porque es la apetencia más preciada del investigador, esta condición y apetencia solo se cumpliría en una minúscula porción de los investigadores de la sociología. ¿Es que en la sociología colombiana no ha cundido lo suficiente el discurso productivista, o es que estos resultados comunican algún movimiento enérgico de resistencia a lo hegemónico? 
Tabla 6. Promedio de artículos por ańo

\begin{tabular}{lcccc}
\hline & Frecuencia & Porcentaje & Porcentaje válido & Porcentaje acumulado \\
\hline Hasta 0,5 & 51 & 39,5 & 40,8 & 40,8 \\
$0,51-0,75$ & 18 & 14,0 & 14,4 & 55,2 \\
$0,76-1.00$ & 25 & 19,4 & 20,0 & 75,2 \\
$1,01-1,25$ & 7 & 5,4 & 5,6 & 80,8 \\
$1,26-1,50$ & 8 & 6,2 & 6,4 & 87,2 \\
$1,51-2,00$ & 11 & 8,5 & 8,8 & 96,0 \\
$2,01-$ HI & 5 & 3,9 & 4,0 & 100,0 \\
No hay dato & 4 & 3.1 & & \\
\hline Total & 129 & 100,0 & 100,0 & \\
\hline
\end{tabular}

Como hemos expresado, hay una prescripción de realización científica o académica muy típica en el proyecto dominante de ciencia y ciencias sociales del presente. Se determina ahí que la escala axiológica del investigador contemporáneo conjugue el apremio de la publicación abundante y vehemente con el valor de la publicación en diferentes casas editoriales. Hay o habría que "evitar la endogamia», pues es algo mal visto (Masías, 2017). Con revistas distintas de publicación tratamos, en las investigaciones del programa, de aprehender la condición actual de estas expectativas axiológicas que reflejan realización, en los términos de nuestra propia perspectiva. En la tabla 2 se constata un promedio de 6,48 revistas distintas, en un plano de mucha desigualdad. Ya hemos indicado que es difícil interpretar el sentido de esta variable por el desafío que presenta determinar la línea base de una situación endogámica. Sea como fuere, serían bastantes los sociólogos que diversifican menos la divulgación de sus creaciones intelectuales, como queda claro en la tabla 7: el $54,3 \%$ no llega al promedio, solo $27,9 \%$ lo supera.

La tabla 8 nos ubica en el contexto de otra manifestación de la realización, como la divulgación del pensamiento o la investigación en la forma de capítulos de libro. Ahí se observa que 70,6\% tiene en su haber hasta cinco capitulos de libro. La sociología no escapa de unas tendencias que hemos venido constatando: los grandes realizadores de capítulos también son escasos. 
Tabla 7. Revistas distintas de publicación

\begin{tabular}{lcccc}
\hline & Frecuencia & Porcentaje & Porcentaje válido & Porcentaje acumulado \\
\hline Hasta 2 & 39 & 30,2 & 30,2 & 30,2 \\
$3-5$ & 31 & 24,0 & 24,0 & 54,3 \\
$6-8$ & 23 & 17,8 & 17,8 & 72,1 \\
$9-11$ & 10 & 7,8 & 7,8 & 79,8 \\
$12-\mathrm{Hi}$ & 26 & 20,2 & 20,2 & 100,0 \\
\hline Total & 129 & 100,0 & 100,0 & \\
\hline
\end{tabular}

Tabla 8. Capítulos de libros publicados

\begin{tabular}{lcccc}
\hline & Frecuencia & Porcentaje & Porcentaje válido & Porcentaje acumulado \\
\hline 0 a 5 & 89 & 69,0 & 70,6 & 70,6 \\
$6-10$ & 21 & 16,3 & 16,7 & 87,3 \\
$11-15$ & 9 & 7,0 & 7,1 & 94,4 \\
$16-20$ & 3 & 2,3 & 2,4 & 96,8 \\
$21-25$ & 1 & 0,8 & 0,8 & 97,6 \\
$26-30$ & 2 & 1,6 & 1,6 & 99,2 \\
31 a más & 1 & 0,8 & 0,8 & 100,0 \\
No hay dato & 3 & 2,3 & & \\
\hline Total & 129 & 100,0 & 100,0 & \\
\hline
\end{tabular}

En correspondencia con los intereses del programa, siguiendo su espíritu comparativo y replicador de su metodología, y en consonancia con el objetivo de conocer el estado de la disciplina, en esta investigación se exploraron las ligazones entre elaboración de libros y de artículos. Tal como lo concebimos, es una época para la ciencia y las ciencias sociales en que se da una pugna entre un tipo de realización y otro. Están apareciendo, como formas antagónicas de realizar y comunicar lo que se hace, formas excluyentes que se viven dilemáticamente. Hacer libros, escribirlos, riñe con la elaboración y publicación de artículos. Se ha creado y fomentado todo un estado de ánimo sobre que "producir» libros es menos rentable y productivo, menos significativo en el conjunto de la obra del investigador que ser un investigador articulista. En todo caso, son prácticas intelectuales que expresan instituciones, formas y grados de institucionalización y en tanto tales sirven bastante en la determinación del estado de una disciplina, como la sociología, entendida esta como un campo (Bourdieu, 2003, 2005).

Según la tabla 2, hay un promedio de 2,67 libros publicados por sociólogo, y una desviación estándar de 3,519 que declararía una inmensa disparidad. La comunidad 
de la sociología sería un colectivo más tendiente a la realización de artículos, en tanto que el promedio de los primeros $(10,02)$ es casi cuatro veces más que de los segundos, los libros.

Las siguientes cuatro tablas $(9,10,11$ y 12$)$ revelarían los mismos patrones de realización hallados en el análisis ejecutado hasta aquí, para el caso de la sociología colombiana: un gran sector con poco en su haber y un pequeñísimo sector con mucho que ostentar. Ahora bien, se puede ser más prolijo si se detiene más en la tabla 10, ya que el $69 \%$ de los sociólogos escribe medio libro por ańo y solo $8,7 \%$ escribe de un libro a más por año. En la tabla 11 vale posar la mirada en el 48,8\% que únicamente ha publicado hasta cinco artículos, y en la tabla 12, el 74,6\% que solamente publica hasta un artículo por año.

Tabla 9. Libros publicados

\begin{tabular}{lcccc}
\hline & Frecuencia & Porcentaje & Porcentaje válido & Porcentaje acumulado \\
\hline Hasta & 64 & 49,6 & 49,6 & 49,6 \\
$2-3$ & 30 & 23,3 & 23,3 & 72,9 \\
$4-5$ & 12 & 9,3 & 9,3 & 82,2 \\
$6-7$ & 11 & 8,5 & 8,5 & 90,7 \\
5,00 & 12 & 9,3 & 9,3 & 100,0 \\
\hline Total & 129 & 100,0 & 100,0 & \\
\hline
\end{tabular}

Tabla 10. Promedio de libros por año

\begin{tabular}{lcccc}
\hline & Frecuencia & Porcentaje & Porcentaje válido & Porcentaje acumulado \\
\hline Hasta 0,5 & 87 & 67,4 & 69,0 & 69,0 \\
$0,51-0,75$ & 17 & 13,2 & 13,5 & 82,5 \\
$0,76-1,00$ & 11 & 8,5 & 8,7 & 91,3 \\
$1,00-$ HI & 11 & 8,5 & 8,7 & 100,0 \\
No hay dato & 3 & 2,3 & & \\
\hline Total & 129 & 100,0 & 100,0 & \\
\hline
\end{tabular}


Tabla 11. Artículos publicados

\begin{tabular}{lcccc}
\hline & Frecuencia & Porcentaje & Porcentaje válido & Porcentaje acumulado \\
\hline 0 a 5 & 62 & 48,1 & 48,8 & 48,8 \\
$6-10$ & 23 & 17,8 & 18,1 & 66,9 \\
$11-15$ & 15 & 11,6 & 11,8 & 78,7 \\
$16-20$ & 11 & 8,5 & 8,7 & 87,4 \\
$21-25$ & 7 & 5,4 & 5,5 & 92,9 \\
$26-30$ & 2 & 1,6 & 1,6 & 94,5 \\
31 a más & 7 & 5,4 & 5,5 & 100,0 \\
No hay dato & 2 & 1,6 & & \\
\hline Total & 129 & 100,0 & 100,0 & \\
\hline
\end{tabular}

Tabla 12. Promedio de artículos por año: intervalo

\begin{tabular}{lcccc}
\hline & Frecuencia & Porcentaje & Porcentaje válido & Porcentaje acumulado \\
\hline Hasta 0,5 & 51 & 39,5 & 40,5 & 40,5 \\
$0,51-0,75$ & 18 & 14,0 & 14,3 & 54,8 \\
$0,76-1.00$ & 25 & 19,4 & 19,8 & 74,6 \\
$1,01-1,25$ & 7 & 5,4 & 5,6 & 80,2 \\
$1,26-1,50$ & 8 & 6,2 & 6,3 & 86,5 \\
$1,51-2,00$ & 11 & 8,5 & 8,7 & 95,2 \\
$2,01-$ HI & 5 & 3,9 & 4,0 & 99,2 \\
No hay dato & 4 & 3,1 & & \\
\hline Total & 129 & 100,0 & & \\
\hline
\end{tabular}

\section{EXPLICANDO EL ESTADO DE LA REALIZACIÓN CIENTÍfICA Y EL ESTADO DE LA SOCIOLOGÍA}

¿Qué explica este estado de realización científica en la sociología colombiana, que remite a un estado de esta disciplina como microcosmos social, con significativas fragmentaciones? Como estaba previsto en el diseño de la investigación, pero también replicando las otras investigaciones del programa, para comparar, se aplicó el «análisis bivariable dicotómico». La medida de asociación elegida fue el llamado Coeficiente de Contingencia (CC en las tablas). Las tablas de contingencia que pasamos a analizar son de las asociaciones que resultaron significativas al 0,05\%; no obstante, se mencionarán ciertos cruces no significativos porque, si bien no apoyan una verificación, lo hacen como elementos de falsación, en una concepción popperiana de la ciencia. 


\section{SOCIÓLOGAS Y SOCIÓLOGOS: LA DESIGUALDAD DE GÉNERO}

El papel del género, como variable explicativa, sería un tanto paradójico. Precisamente, en cuanto a número total de productos, no serían mucho menos las mujeres que pertenecen a la categoría de los investigadores más realizadores, es decir los que corresponden a la categoría "De once a más productos en total": $41,5 \%$ contra $57,9 \%$ (tabla 13). Hasta podría aceptarse que, en el plano de la obra de conjunto, mujeres y varones logran acumular unos bienes académicos, en general, equiparables. No obstante, esta visión cambia mucho cuando se observa la diferencia de género en la publicación de artículos. En la categoría de más realización, el porcentaje de varones duplica al de mujeres (tabla 14). Similar patrón se manifiesta en la tabla 15, que corresponde a la publicación de capítulos. Vale advertir que, sobre publicación de artículos, promedio de artículos y libros por año, los cruces no resultaron significativos. Ahora bien, si estos datos se examinaran desde una perspectiva productivista, la conclusión hablaría de una menor o nula productividad de las mujeres, interpretación que dejaría escapar la posibilidad de la existencia de unas expectativas y estrategias distintas de realización entre las sociólogas.

Tabla 13. Número de productos en total por género

\begin{tabular}{llccc}
\hline & & Masculino (\%) & Femenino (\%) & Rubro (\%) \\
\hline Número de productos & Hasta diez & 42,1 & 58,5 & 48,8 \\
en total & De once a más & 57,9 & 41,5 & 51,2 \\
\hline Total & & 100,0 & 100,0 & 100,0 \\
\hline CC:, $159 /, 067$ & & & \\
N 129 & & &
\end{tabular}

Tabla 14. Número de artículos publicados por género

\begin{tabular}{llccc}
\hline & & Masculino (\%) & Femenino (\%) & Rubro (\%) \\
\hline Número de artículos & Hasta diez & 56,6 & 79,2 & 65,9 \\
publicados & De once a más & 43,4 & 20,8 & 34,1 \\
\hline Total & & 100,0 & 100,0 & 100,0 \\
\hline
\end{tabular}

CC:, $229 /, 008$

N 129 
Tabla 15. Número de capítulos de libro publicados por género

\begin{tabular}{llccc}
\hline & & Masculino (\%) & Femenino (\%) & Rubro (\%) \\
\hline $\begin{array}{l}\text { Número de } \\
\begin{array}{l}\text { capítulos de libro } \\
\text { publicados }\end{array}\end{array}$ & Hasta tres & 50,0 & 67,9 & 57,4 \\
\hline Total & De cuatro a más & 50,0 & 32,1 & 42,6 \\
\hline
\end{tabular}

CC:, $176 /, 043$

N 129

\section{UNA SOCIOLOGÍA DESCENTRALISTA Y DESCENTRALIZADA}

Esta sección es una glosa sobre unos resultados que, para la realidad de la sociología, no se hicieron presentes. Como ocurría en las investigaciones anteriores, la variable lugar de trabajo (Bogotá, Medellín, Cali, etc.) resultaba contundente para dar cuenta de un estado disciplinar de fragmentación y desigualdad académica respecto de las regiones en Colombia. Es conocido el peso de la espacialidad en el país. En efecto, trabajar en Bogotá, en una universidad bogotana, es, al parecer, un enorme factor de desigualdad de realización y, por ende, de desigualdad social en la academia. Para el contexto de la sociología, ninguno de los cruces ensayados dio resultados estadísticamente significativos. Este resultado amerita más investigación, máxime cuando se sabe que la sociología colombiana habría gozado de la misma fortaleza en las distintas partes del país donde ha habido esta disciplina.

\section{REALIZACIÓN Y TOP ACADÉMICO SOCIOLÓGICO}

Poco se cuestiona el impacto de la «institución» en la realización del investigador social. «Institución» equivale a todo un soporte que están en capacidad de brindar, y que brindan, las instituciones académicas y científicas para el desarrollo intelectual. No son otra cosa que unas condiciones favorables para la realización. Los rankings en boga reflejan lo que significa pertenecer a una institución académica prestigiosa y poderosa económicamente que a otra que lo sea menos. La variable o indicador institución académica de trabajo posee dos categorías: del top, que equivale a trabajar o ser de la planta de en cualquiera de estas tres universidades: Universidad de los Andes, Universidad Nacional de Colombia y Universidad de Antioquia ${ }^{4}$, y Todas las demás.

\footnotetext{
4 Hicimos este ejercicio para hacer un juicio de la visión de medios de comunicación y del estado sobre la realidad de las universidades en el país. Estas universidades aparecen ocupando, en efecto, los primeros lugares de los rankings.
} 
Según la tabla 16, se desprendería que es en las universidades del top donde se alojaría el mayor patrimonio de capital académico, patrón documentado también en las otras investigaciones del programa. El sociólogo empleado en una de esas universidades gozaría de condiciones propicias para una realización académica más plena. No sobra comentar que, en esas universidades, en efecto, habría mucha más "producción y productividad» académica, una situación que, hilando, llevaría a pensar en la existencia real de una élite en la sociología colombiana.

Tabla 16. Número de productos en total por institución académica de trabajo: universidad top

\begin{tabular}{llccc}
\hline & & Todas las demás (\%) & Del top (\%) & Rubro (\%) \\
\hline \multirow{2}{*}{$\begin{array}{l}\text { Número de } \\
\text { productos en total }\end{array}$} & Hasta diez & 63,1 & 34,4 & 48,8 \\
& De once a más & 36,9 & 65,6 & 51,2 \\
\hline \multirow{2}{*}{ Total } & & $\mathbf{1 0 0 , 0}$ & $\mathbf{1 0 0 , 0}$ & $\mathbf{1 0 0 , 0}$ \\
\hline
\end{tabular}

CC:, $276 /, 001$

N 129

Las tablas que siguen, si bien cada una manifiesta una problemática particular a tener en consideración, fortificarían esta sensación sobre el peso explicativo, pero también sobre la realidad que representaría ser sociólogo de unas y no de otras universidades. Entre las tablas 17 a 20, lo que hay es una pauta como sigue: los porcentajes de sociólogos que pertenecen al top y que pertenecen a las categorías de mayor realización, o casi duplican en alguna variable o indicador, o triplican al porcentaje de las otras universidades.

Tabla 17. Número de libros publicados por institución académica de trabajo: universidad top

\begin{tabular}{llccc}
\hline & & Todas las demás (\%) & Del top (\%) & Rubro (\%) \\
\hline Número de libros & Hasta uno & 61,5 & 37,5 & 49,6 \\
publicados & De dos a más & 38,5 & 62,5 & 50,4 \\
\hline Total & & 100,0 & 100,0 & 100,0 \\
\hline
\end{tabular}

CC: $234 /, 006$

N 129 
Tabla 18. Promedio de libros por ańo por institución académica de trabajo: universidad top

\begin{tabular}{llccc}
\hline & & Todas las demás (\%) & Del top (\%) & Rubro (\%) \\
\hline Promedio de libros & Hasta 0,5 & 86,2 & 53,1 & 69,8 \\
por ańo & De 0,51 a más & 13,8 & 46,9 & 30,2 \\
\hline \multirow{2}{*}{ Total } & & 100,0 & 100,0 & 100,0 \\
\hline
\end{tabular}

CC: ,338 / ,000

N 129

Tabla 19. Número de artículos publicados por institución académica de trabajo: universidad top

\begin{tabular}{llccc}
\hline & & Todas las demás (\%) & Del top (\%) & Rubro (\%) \\
\hline Número de artículos & Hasta diez & 78,5 & 53,1 & 65,9 \\
publicados & De once a más & 21,5 & 46,9 & 34,1 \\
\hline Total & & 100,0 & 100,0 & 100,0 \\
\hline
\end{tabular}

CC:, $258 /, 002$

N 129

Tabla 20. Número de capítulos de libro publicados por institución académica de trabajo: universidad top

\begin{tabular}{llccc}
\hline & & Todas las demás (\%) & Del top (\%) & Rubro (\%) \\
\hline $\begin{array}{l}\text { Número de capítulos } \\
\text { de libro publicados }\end{array}$ & Hasta tres & 69,2 & 45,3 & 57,4 \\
\cline { 2 - 5 } & De cuatro a más & 30,8 & 54,7 & 42,6 \\
\hline \multirow{2}{*}{ Total } & 100,0 & 100,0 & 100,0 \\
\hline
\end{tabular}

CC: 2357,006

N 129

\section{LA REALIZACIÓN Y EL GRADO DE DOCTOR}

La «institución», como se acaba de ver, y el «doctorado» ahora, son dos realidades por mirar más allá de unas formalidades. Así lo teníamos previsto en el diseño de la investigación. Son "formas», a la manera de Simmel. Son unas manifestaciones sociológicas pletóricas de significados sobre lo que es el mundo de la ciencia contemporánea, y en particular de los sociólogos. Lo que se palpa en las tablas que siguen, contra lo que se tiende a pensar, es que tener un doctorado, en el contexto de las llamadas exigencias actuales (de producción y productividad), faculta a mayores posibilidades y probabilidades de realización y no solo a la consecución de unos méritos para ascender en una carrera académica. Habría una complicidad 
entre lo que forma un doctorado y el tipo de presiones que tienen que ver con las concepciones, axiología y orientaciones realizativas que se fomentan y premian más en el momento presente.

Las tablas 21 a 25 evidenciarían más rotundamente (que con relación a la institución académica de trabajo) la diferencia que hace tener o no doctorado en la actualidad entre los sociólogos. Además, a despecho de las otras variables explicativas puestas en juego para todas las variables dependientes, con esta casi todos los cruces son significativos.

Tabla 21. Número de productos en total por posesión de doctorado

\begin{tabular}{llccc}
\hline & & $\begin{array}{c}\text { Sin doctorado } \\
(\%)\end{array}$ & $\begin{array}{c}\text { Con doctorado } \\
(\%)\end{array}$ & $\begin{array}{c}\text { Rubro } \\
(\%)\end{array}$ \\
\hline Número de & Hasta diez & 66,7 & 31,8 & 48,8 \\
productos en total & De once a más & 33,3 & 68,2 & 51,2 \\
\hline Total & & 100,0 & 100,0 & 100,0 \\
\hline
\end{tabular}

CC:, $329 /, 000$

N 129

Tabla 22. Promedio de libros por año por posesión de doctorado

\begin{tabular}{llccc}
\hline & & $\begin{array}{c}\text { Sin doctorado } \\
(\%)\end{array}$ & $\begin{array}{c}\text { Con doctorado } \\
(\%)\end{array}$ & $\begin{array}{c}\text { Rubro } \\
(\%)\end{array}$ \\
\hline \multirow{2}{*}{$\begin{array}{l}\text { Promedio de } \\
\text { libros por año }\end{array}$} & Hasta 0,6 libros por año & 81,7 & 65,2 & 73,0 \\
& De 0,61 a más libros por año & 18,3 & 34,8 & 27,0 \\
\hline \multirow{2}{*}{ Total } & & 100,0 & 100,0 & 100,0 \\
\hline
\end{tabular}

CC:, $183 /, 037$

N 126

Tabla 23. Número de artículos publicados por posesión de doctorado

\begin{tabular}{llccc}
\hline & & $\begin{array}{c}\text { Sin doctorado } \\
(\%)\end{array}$ & $\begin{array}{c}\text { Con doctorado } \\
(\%)\end{array}$ & $\begin{array}{c}\text { Rubro } \\
(\%)\end{array}$ \\
\hline Número de artículos & Hasta diez & 84,1 & 48,5 & 65,9 \\
publicados & De once a más & 15,9 & 51,5 & 34,1 \\
\hline \multirow{2}{*}{ Total } & & 100,0 & 100,0 & 100,0 \\
\hline
\end{tabular}

CC:, $352 /, 000$

N 129 
Tabla 24. Promedio de artículos por año por posesión de doctorado

\begin{tabular}{llccc}
\hline & & $\begin{array}{c}\text { Sin } \\
\text { doctorado } \\
(\%)\end{array}$ & $\begin{array}{c}\text { Con } \\
\text { doctorado } \\
(\%)\end{array}$ & $\begin{array}{c}\text { Rubro } \\
(\%)\end{array}$ \\
\hline $\begin{array}{l}\text { Promedio de } \\
\text { artículos por año }\end{array}$ & Menos de un artículo por año & 80,0 & 59,1 & 69,0 \\
\hline Total & Eno y más artículos por año & 20,0 & 40,9 & 31,0 \\
\hline
\end{tabular}

CC:, $220 /, 011$

N 126

Tabla 25. Número de capítulos de libro publicados por posesión de doctorado

\begin{tabular}{llccc}
\hline & & $\begin{array}{c}\text { Sin doctorado } \\
(\%)\end{array}$ & $\begin{array}{c}\text { Con doctorado } \\
(\%)\end{array}$ & $\begin{array}{c}\text { Rubro } \\
(\%)\end{array}$ \\
\hline $\begin{array}{l}\text { Número de capítulos } \\
\text { de libro publicados }\end{array}$ & Hesta tres & 73,0 & 42,4 & 57,4 \\
\hline Total & De cuatro a más & 27,0 & 57,6 & 42,6 \\
\hline
\end{tabular}

CC: $295 /, 000$

N 129

\section{No TODOS LOS DOCTORADOS SON IGUALES}

Efectuamos un análisis más cuidadoso del peso explicativo del fenómeno "doctorado». Este análisis se practicó posando la mirada en la región o país donde este se consiguió. El indicador se denomina región intelectual del doctorado, con dos categorías: centros metropolitanos, que es el conjunto conformado por doctorados realizados en Estados Unidos, Alemania, Francia o Inglaterra, y periferia, que son todos los demás doctorados. Cuando aplicamos este como variable independiente de las de realización académica, ninguna de las asociaciones fue significativa: un resultado inesperado en comparación con los estudios anteriores. Además de investigar más a fondo el hallazgo, llama la atención que, en el caso de la sociología, sería lo mismo, en cuanto a eficiencia y eficacia de realización, que se haya conseguido el doctorado en los llamados centros que en la periferia.

La única correlación que arrojó significación estadística es la que conjuga la región intelectual de las maestrías de nuestros sociólogos con el promedio de artículos por año (tabla 26). 
Tabla 26. Promedio de artículos por año por región Intelectual: maestría

\begin{tabular}{|c|c|c|c|c|}
\hline & & $\begin{array}{l}\text { Periferia } \\
(\%)\end{array}$ & $\begin{array}{c}\text { Centros } \\
\text { metropolitanos (\%) }\end{array}$ & $\begin{array}{c}\text { Rubro } \\
(\%)\end{array}$ \\
\hline \multirow{2}{*}{$\begin{array}{l}\text { Promedio de } \\
\text { artículos por año }\end{array}$} & $\begin{array}{l}\text { Menos de un artículo } \\
\text { por ańo }\end{array}$ & 73,3 & 51,4 & 66,4 \\
\hline & $\begin{array}{l}\text { Entre uno y más } \\
\text { artículos por año }\end{array}$ & 26,7 & 48,6 & 33,6 \\
\hline Total & & 100,0 & 100,0 & 100,0 \\
\hline
\end{tabular}

CC:, $211 /, 024$

N 110

\section{SOCIÓLOGOS NETOS VERSUS SOCIÓLOGOS HÍBRIDOS}

Cerramos este estudio analizando el peso explicativo de una variable que, en las otras investigaciones del programa, no mostró significado alguno. Se trata de disciplina afin, una expresión para significar un fenómeno existente en el mundo de las ciencias sociales. Lo que quiere denotar es la presencia de un sector de académicos con un proceso o trayectoria de formación en una misma disciplina, y otro que combina la formación de sociólogo con otras disciplinas u otros campos del saber. Estarían, entonces, los sociólogos de pregrado, de maestría y de doctorado, a quienes denominamos sociólogos netos, junto con los híbridos, aquellos miembros del colectivo sociológico que combinaron estudios en su proceso de formación.

Lo que se presenta en las últimas cinco tablas (tablas 27 a 31) es un mismo acontecimiento: la proporción de los híbridos siempre es mayor en las categorías de más realización científica en casi todos los indicadores que miden esta cuestión. Es decir, los híbridos se posicionan mejor en cuanto a realización, lo que es lo mismo que decir que tienen un mayor potencial de realización.

Conjeturando sobre esta situación con la lógica del régimen académico imperante, ser híbrido constituiría una condición que favorece la producción y productividad. Por el contrario, la "pureza» disciplinar pondría en condiciones de inferioridad, en términos de eficacia en la economía política contemporánea de la ciencia o del conocimiento. ¿Qué competencias incluye, integra e implica la hibridez? Por oposición, ¿qué haría al «sociólogo total» o neto menos competente en estas lides?

No obstante, de ser cierto este fenómeno, su trascendencia podría ir más allá del mero asunto de la capacidad de producir más o del establecimiento de aquellos factores que explicarían más y mejor la realización. Estamos sugiriendo sus implicancias en la discusión del estado de la disciplina y en la cuestión de la fragmentación y desigualdad social en la sociología y en las ciencias sociales. Pues, ¿qué 
quiere decir que, en una disciplina en particular, no sea en sus más puros exponentes en quienes recaiga el dinamismo cognitivo e investigativo de tal ciencia? Cuando hablamos del estado de la sociología y sus aportes, ¿en verdad de quiénes estamos hablando? ¿Qué sujetos forjan la sociología? ¿Son sociólogos? Y si queremos integrar el elemento valorativo, ¿está bien que no sean estos sujetos «tan» sociólogos?

Tabla 27. Número de productos en total por disciplina afín

\begin{tabular}{llccc}
\hline & & Sí (\%) & No (\%) & Rubro (\%) \\
\hline Número de & Hasta diez & 64,9 & 42,7 & 49,2 \\
productos en total & De once a más & 35,1 & 57,3 & 50,8 \\
\hline Total & & 100,0 & 100,0 & 100,0 \\
\hline
\end{tabular}

CC: , $198 /$, 023

N 126

Tabla 28. Número de libros publicados por disciplina afín

\begin{tabular}{llccc}
\hline & & Sí & No & Rubro (\%) \\
\hline Número de libros & Hasta uno & 67,6 & 42,7 & 50,0 \\
publicados & De dos a más & 32,4 & 57,3 & 50,0 \\
\hline Total & & 100,0 & 100,0 & 100,0 \\
\hline
\end{tabular}

CC:, $221 /, 011$

N 126

Tabla 29. Número de artículos publicados por disciplina afín

\begin{tabular}{llccc}
\hline & & Sí (\%) & No (\%) & Rubro (\%) \\
\hline Número de artículos & Hasta diez & 81,1 & 60,7 & 66,7 \\
publicados & De once a más & 18,9 & 39,3 & 33,3 \\
\hline Total & & 100,0 & 100,0 & 100,0 \\
\hline
\end{tabular}

CC:, $193 /, 027$

N 126

Tabla 30. Promedio de artículos por año por disciplina afín

\begin{tabular}{llccc}
\hline & & Sí (\%) & No (\%) & Rubro (\%) \\
\hline Promedio de & Menos de un artículo por año & 89,2 & 60,9 & 69,4 \\
artículos por año & Entre uno y más artículos por año & 10,8 & 39,1 & 30,6 \\
\hline Total & & 100,0 & 100,0 & 100,0 \\
\hline
\end{tabular}

CC:, $270 /, 002$

N 124 
Tabla 31. Número de capítulos de libro publicados por disciplina afín

\begin{tabular}{llccc}
\hline & & Sí (\%) & No (\%) & Rubro (\%) \\
\hline Número de capítulos & Hasta tres & 73,0 & 51,7 & 57,9 \\
de libro publicados & De cuatro a más & 27,0 & 48,3 & 42,1 \\
\hline Total & & 100,0 & 100,0 & 100,0 \\
\hline
\end{tabular}

CC:, $193 /, 027$

N 126

\section{REFLEXIONES FINALES}

El interés de nuestro trabajo no ha sido evidenciar lo poco o mucho que los investigadores sociólogos producen: para ello bastaría con recurrir a los datos existentes en los informes que los organismos de medición nacional realizan con periodicidad. Lo que ha interesado tratar es la constitución social de la sociología, vista como un microcosmos particular. Para este cometido se puso en práctica una perspectiva de realización científica. Una aproximación en clave de realización científica sugiere un mundo social sociológico fragmentado, un estado fragmentado de la sociología colombiana.

Por su parte, la perspectiva puesta en juego permite reinterpretar las formas usuales de manejo de los datos de realización científica. Es el caso de la situación de las sociólogas colombianas, que podrían ser vistas como parte de los sectores menos productivos o improductivos, pues su participación resulta escasa o marginal respecto de los indicadores privilegiados en la medición del rendimiento exitoso científico. En lugar de poca productividad o improductividad, podría estar ocurriendo que se desconoce lo que las sociólogas entienden, pretenden y esperan de su labor investigativa. Por decirlo en nuestros términos, se desconocen sus expectativas de realización.

La sociología parece ser un colectivo donde se reproducen las lógicas de concentración de otros ámbitos de la vida social: es un colectivo profundamente desigual desde el punto de vista de la realización científica. Muy pocos concentrarían una obra académica valorada como excepcional, por decirlo así, muy convencionalmente. La realización científica, vista como bien, no sería un bien de goce amplio. Si esta visión fuese correcta o cierta, la pregunta es, entonces, en qué sujeto o sector ha recaído y recae la llamada producción investigativa creciente de la sociología, la variada producción bibliográfica, la mayor interacción de esta disciplina con otras ciencias y la producción de ideas y el establecimiento de la institucionalidad sociológica.

A expensas de los resultados de esta investigación, habría que prestar más atención a la tesis sobre la existencia de sociologías, en plural, en el país: la visión de las 
sociologías diversas, en lugar de una monolítica disciplina que va solidificándose cada vez más. También, coherentes con la unidad de análisis de este trabajo —el investigador de la sociología- habría que discutir si los avances globales de la disciplina los serían igualmente en el plano individual, de las personas.

Abiertas están las nuevas pistas de reflexión e investigación sobre nuestra disciplina.

\section{REFERENCIAS}

Arecibia, R. y Moya, F. (2008). La evaluación de la investigación científica: una aproximación teórica desde la cienciometría. ACIMED, 17(4), 1-27.

Borrego, Á. y Urbano, C. (2006). La evaluación de revistas científicas en Ciencias Sociales y Humanidades. Información, Cultura y Sociedad, 14, 1-21.

Bourdieu, P. (2003). El oficio de cientifico. Ciencia de la ciencia y reflexividad. Barcelona: Anagrama.

Bourdieu, P. (2005). Los usos sociales de la ciencia. Buenos aires: Nueva Visión.

Camacho, A. y Hernández, J. (1990). La investigación sociológica en Colombia en la década de los ochentas, un intento de evaluación. Recuperado de https://ideas.repec.org/p/ col/000149/003983.html

Cubides, F. (2011). La sociología colombiana de cara al siglo XXI. Colombia, Boletín Cultural y Bibliográfico, XLV, 135-49.

Cubides, F. (1998). La sociología en Colombia: demandas y tribulaciones. Revista de Estudios Sociales, 1, 1-7. https://doi.org/10.7440/res1.1998.01

De Sousa Santos, B. (2003). Crítica de la razón indolente. Contra el desperdicio de la indolencia. Bilbao: Desclée.

De Sousa Santos, B. (2010). Para descolonizar Occidente. Más allá del pensamiento abismal. Buenos Aires: Clacso.

Forero, F. (2015). Cientificos sociales y humanistas en la nueva convocatoria de medición de grupos de COLCIENCIAS. Recuperado de http://palabrasalmargen.com/index.php/ articulos/nacional/item/cientificos-sociales-y-humanistas-en-la-nueva-convocatoria-de-medicion-de-grupos-de-colciencias

García, N. (2010). La sociedad sin relato. Antropología y estética de la inminencia. Buenos Aires: Katz.

Gill, R. (2014). Academics, Cultural Workers and Critical Labour Studies. Journal of Cultural Economy, 7(1), 12-30. https://doi.org/10.1080/17530350.2013.861763

Latour, B. (2001). La esperanza de Pandora. Ensayos sobre la realidad de los estudios de la ciencia. Barcelona: Gedisa.

Latour, B. (2012). Cogitamus. Seis cartas sobre las humanidades cientificas. Buenos Aires: Paidos.

Lowy, M. (2000). ¿Qué es la sociología del conocimiento? México, D. F.: Fontamara.

Masias, R. (2012). El libro, el ensayismo y las ciencias sociales en un mundo global y poscolonial. Revista Relaciones Internacionales, 20, 175-182. 
Masías, R. (2013). El investigador social contemporáneo y las Ciencias Sociales a través de sus libros. Historia y espacio, 40, 168-190.

Masías, R. (2014). Los investigadores sociales en Colombia: producción, productividad y diferenciación social. Revista Colombiana de Sociología, 37(1), 123-156.

Masías, R. (2017). Los investigadores de la ciencia política en Colombia. Realización científica y desigualdad social. Estudios Políticos, 51, 128-157.

Merton, R. (2002). Teoría y estructura sociales. México, D. F.: Fondo de Cultura Económica.

Navarrete, S. (2015). Colciencias y los humanistas, en orillas diferentes. El Espectador, p. 1. Recuperado de http://www.elespectador.com/noticias/nacional/ colciencias-y-los-humanistas-orillas-diferentes-articulo-545541

Segura, N. y Camacho, A (1999). En los cuarenta años de la sociología colombiana. Revista de estudios sociales, 4, 23-35.

Vélez, G., Gómez, H., Úsuga, A. y Vélez-Trujillo, M. (2014). Diversidad y reconocimiento de la producción académica en los sistemas de evaluación de la investigación en Colombia. Revista Española de Documentación Cientifica, 37(3), 1-8. https://doi. org/10.3989/redc.2014.3.1133

Vink, D. (2015). Ciencias y sociedad. Sociologia del trabajo científico. Barcelona: Gedisa. 